\title{
“Kill-TB” Drug Reminder Mobile Application for Tuberculosis Patients at Bengaluru, India: Effectiveness and Challenges
}

\author{
Sharath Burugina Nagaraja ${ }^{1}$, Naveen Kotur ${ }^{1 *}$, Niranjan Murthy ${ }^{1}$, \\ Shilpashree Madhava Kunjathur ${ }^{2}$, Nanda Sappandi ${ }^{3}$, \\ Deepak Murthy3 ${ }^{3}$, Arundathi Das ${ }^{4}$, Anil Singarajipur ${ }^{5}$ \\ ${ }^{1}$ Employee's State Insurance Corporation Medical College and Post Graduate Institute of Medical Sciences and Research, \\ Bengaluru, India \\ ${ }^{2}$ BGS Global Institute of Medical Sciences, Bengaluru, India \\ ${ }^{3}$ Bangalore Medical College and Research Institute, Bengaluru, India \\ ${ }^{4}$ District Tuberculosis Office, Bengaluru, India \\ ${ }^{5}$ State Tuberculosis Office, Bengaluru, India \\ Email: sharathbn@yahoo.com, *naveenskotur@gmail.com
}

How to cite this paper: Nagaraja, S.B., Kotur, N., Murthy, N., Kunjathur, S.M., Sappandi, N., Murthy, D., Das, A. and Singarajipur, A. (2020) “Kill-TB” Drug Reminder Mobile Application for Tuberculosis Patients at Bengaluru, India: Effectiveness and Challenges. Journal of Tuberculosis Research, 8, 1-10.

https://doi.org/10.4236/jtr.2020.81001

Received: December 6, 2019

Accepted: December 31, 2019

Published: January 3, 2020

Copyright $\odot 2020$ by author(s) and Scientific Research Publishing Inc. This work is licensed under the Creative Commons Attribution International License (CC BY 4.0).

http://creativecommons.org/licenses/by/4.0/ (c) (i) Open Access

\begin{abstract}
Background: Tuberculosis remains a major public health problem in India. Newer digital technologies for treatment adherence have been tested for diseases with chronic medication. A mobile application (Kill-TB) that provides reminder to TB patients through alarm was tested for its effectiveness and challenges under programmatic settings. Methods: A cohort of TB patients initiated on treatment with and without mobile application at Bangalore city during January-March 2019 was observed for missed doses during their treatment period. Results: A total of 694 patients (347 patients with and without mobile application) were enrolled for the study. Among the patients with and without mobile application 1) loss to follow-up was $2 \%$ and $8 \%, 2$ ) non-adherence rate to TB treatment was 1.5 and 3.3 per 100 person-months, 3) treatment success rate was $89 \%$ and $81 \%$. Conclusion: The TB drug reminder mobile application benefits the patient's treatment adherence and paves way for improved treatment outcomes despite the challenges in implementation. The programme should leverage mobile technologies as an additional monitoring tool to achieve the target of End-TB by 2025 .
\end{abstract}

\section{Keywords}

Kill-TB, Tuberculosis, India, Mobile Application 


\section{Introduction}

Globally, tuberculosis (TB) continues to be the major public health problem with 10 million people developing TB and 1.6 million dying from the disease [1]. India is one of the high TB burden countries and accounts for $27 \%$ of global TB burden despite the efforts of Revised National TB Control Programme (RNTCP) since 1997 [1]. The programme has achieved the treatment success rate of 79\% $85 \%$ and loss to follow-up of $8 \%-10 \%$ in the last five years [2].

Adherence to tuberculosis treatment is a challenge to patients because of the complexity, longer treatment duration and modest tolerability. The low treatment adherence has the possibility of poorer treatment outcomes like relapse or treatment failure leading to the amplification of drug resistance. Apart from the directly observed treatment (DOT), many e-health strategies have been adopted by different national TB programmes to monitor the treatment adherence with different degrees of success [3]. These strategies include short messaging services, giving missed calls, ingestible sensors, digital pill boxes, smart or mobile phone-based technologies and video observed therapy [4] [5] [6] [7] [8].

In India, under RNTCP, the monitoring of the TB patients initiated on treatment is done by the directly observed treatment (DOT) provider or treatment supporter who is a local person residing nearer to the patient's domicile [9]. The DOT provider is identified by the health worker in consultation with the patient. The adherence to treatment of a TB patient is documented in a TB treatment card maintained by a DOT provider and a card maintained at the primary health center by general health staff. The treatment card at the DOT provider is updated regularly on daily basis, while, the treatment card at the primary health center is updated by the field staff during fortnightly or monthly review meetings at the health facilities. The medical officers or programme managers are not updated in real-time about their patient's deviation from treatment schedule. These delays act as a hindrance for the programme managers to initiate prompt retrieval action and ensure treatment continuation.

The mobile phone users in India have drastically increased to 581 million users when compared to the last decade. The RNTCP had adopted the strategy called 99 DOTS in 2013 wherein the patient had to give a missed call to a number mentioned on the backside of each pill cover [10]. The coverage of this strategy was restricted to a few vulnerable populations and places.

An android mobile application for TB patients called "Kill-TB" was developed to remind the patients through an alarm about their daily drug intake and ensure real-time notifications to the programme manager and RNTCP key staff. We conducted this study to compare the treatment success among patients with and without mobile application and enumerate the challenges encountered during the developmental and implementation process under programmatic settings.

\section{Methods}

\section{1) Study Design}


We conducted a prospective cohort study using routine programmatic data to compare the treatment outcomes among patients with and without mobile application.

\section{2) Settings}

The state of Karnataka is located in southern of India and has a population of 82.74 million. The state has 31 administrative districts and is implementing RNTCP since 1997 [2]. The study was conducted in Bangalore city with a population of 7.8 million, the district has 22 tuberculosis unit (a programmatic management unit for 0.25 million population) and 83 designated microscopy centers (microscopy center to detect TB for 0.1 million population). In the year 2018, the district had notified 11,106 TB patients under public sector and has recorded $81 \%$ treatment success rate [2].

Once the TB patients are diagnosed at the health facilities the patients are initiated on daily regimen therapy for six months after identifying the DOT (Directly observed treatment) provider or treatment supporter who is generally acceptable and accessible to the patient. The DOT provider is provided an honorarium after successful completion of treatment.

\section{3) Study Population, Sample Size and Sampling Technique}

All the adult TB patients diagnosed at three select tuberculosis unit of Bangalore city during the period January-March 2019 were included in the study. The sample size was calculated based on the assumption of treatment success rate among patients with and without mobile application as $80 \%$ and $88 \%$ respectively [11]. It is calculated with $80 \%$ power and $95 \%$ confidence interval and was found to be 331 in each group. Considering the attrition rate of 5\% the total sample size for the study was found to be 694 . The adult TB patients who had android mobile phones with internet connection and are willing to be part of the study formed the intervention group and those patients without android mobile phones or not willing to be part of the study formed the non-intervention group. The patients were enrolled consecutively till the sample size was achieved.

\section{4) Intervention Strategy}

"Drug reminder for TB patients"-The strategy involves reminding the patient for consumption of TB drugs through their android mobile applications. The android mobile phones of the patients remind them about the consumption of individual drugs at a set time through an alarm. The alarm can be stopped once the patient confirms the intake of drug through a button on the mobile phone. The exact time of intake for each of the drugs are recorded in the backhand of a server. The programme manager has access to monitor the drug intake of all the TB patients in the area. If the patient forgets to consume the drugs at a set date and time, a text message is sent automatically to the mobile phones of the DOT provider and patients close contact. Efforts from the DOT provider will be made to retrieve the patient back to treatment. The patients will also be reminded through text messages for timely sputum follow up examination after completion of their intensive phase and continuation phase. 
Standard operating procedure followed for registration of TB patients in the intervention group

Patients are briefed about the study and written informed consent is taken for their participation in the study. The patients NIKSHAY identity number, age, sex, address, type of TB, mobile number of patient and their close contact are taken. The "Kill-TB" mobile application is then downloaded into the patients android mobile and one-time registration is done after entering the patient and treatment details. The patients preferred timings for drug intake shall be set in the patient's phone. Demonstration on usage of the mobile application is provided to the patient by trained personnel. Once the registrations are complete a message regarding the successful registration in mobile application is sent to all the concerned health care personnel's which includes programme manager, senior treatment supervisor, TB health visitor, medical officers TB control, DOT provider and patients assigned contact person. The mobile application will remind the patient about the sputum follow up before two remaining doses at the intensive and continuation phase.

Operational definition for treatment adherence

A TB patient is considered to be treatment adherent, if the patient misses $\leq 2$ doses consecutively in a month during his treatment period.

\section{Development and pilot testing of mobile application}

The mobile application was developed by a private software company specialized in developing health related mobile application. The application was developed during January 2017 to September 2018 after detailed deliberation, pilot testing and fixing the technical software bugs. The field testing of the mobile application was done during October to December 2018.

\section{Sources of data and data variables}

The data for the intervention group was extracted from the electronic data maintained in the electronic database of "Kill-TB" mobile application web based-server for the period January to September 2019. The data for the non-intervention group was extracted from paper-based patients' registers and TB treatment cards at the health facilities. The data variables collected included the clinical and socio-demographic characteristics of patients along with their treatment adherence and treatment outcomes. The data from non-intervention group was entered into EpiData software (version 3.1, EpiData Association, Odense, Denmark) by the investigators. The data exported from the electronic data based and the data entered into EpiData from TB patients' records were merged and a final database was created for data analysis.

\section{Data analysis and statistics}

The electronic format of the data was imported into EpiData Analysis software (version 2.2.2.182, EpiData Association, Odense, Denmark) and was analyzed for descriptive statistics like frequencies, proportions, measures of central tendency (mean) and variation (standard deviation). The treatment non-adherent cases were calculated as per 100 person-months and the rates were compared with intervention and non-intervention group using chi-square test. The p-value 
less than 0.05 was considered as significant.

\section{Ethics approval}

The ethics approval was obtained from the institutional ethics committee of ESIC Medical College and PGIMSR, Bengaluru, India during July 2015. The permission for the conduct of the study was obtained from the RNTCP State TB Office, Karnataka.

\section{Results}

A total of 694 patients were enrolled in this study. The mean age of the study population was 46 years (standard deviation of 17). There were 347 TB patients in the intervention (patients with mobile application) and non-intervention (patients without mobile application) group. The characteristics of the intervention and non-intervention group are shown in Table 1. In both these groups, majority of them were males (73\% and $62 \%$ ) and were microbiologically confirmed cases $(77 \%$ and $72 \%)$. The treatment success was found to be $89 \%$ and $81 \%$ respectively in the intervention and non-intervention group. The loss to follow-up in the intervention group was $2 \%$ when compared to $8 \%$ in the non-intervention group and it was found to be statistically significant. The non-adherence rate to TB treatment was found to 1.5 and 3.3 per 100 person-months respectively among intervention group and non-intervention group and it was found to be statistically significant. The key challenges encountered by the investigators during the software mobile application development and implementation process are shown in Box 1.

Table 1. Characteristics of diagnosed tuberculosis patients in the intervention (with mobile application) and non-intervention (without mobile application) group at the select tuberculosis units of Bengaluru city during January 2019-September 2019 ( $\mathrm{N}=694)$.

\begin{tabular}{cccc}
\hline Characteristics & $\begin{array}{c}\text { Intervention group } \\
(\mathbf{n}=347)\end{array}$ & $\begin{array}{c}\text { Non-intervention group } \\
(\mathbf{n}=347)\end{array}$ & p-value* \\
\hline Age groups (in years) & $\mathbf{n}(\%)$ & $\mathbf{n}(\%)$ & \\
$<25$ & $38(11)$ & $42(12)$ & Ref \\
25 to 34 & $87(25)$ & $66(19)$ & \\
35 to 44 & $66(19)$ & $49(14)$ & $<0.01$ \\
45 to 54 & $66(19)$ & $56(16)$ & \\
55 to 64 & $52(15)$ & $62(18)$ & \\
$>64$ & $38(11)$ & $73(21)$ & \\
Gender & & $215(62)$ & \\
Male & $253(73)$ & $132(38)$ & \\
Female & $94(27)$ & & \\
Diagnosis & & $97(28)$ & \\
Clinical & $80(23)$ & $250(72)$ & \\
Microbiological & $267(77)$ & & \\
\hline
\end{tabular}




\section{Continued}

\begin{tabular}{cccc}
\hline Treatment outcomes & & & \\
Treatment success & $309(89)$ & $281(81)$ & \\
Died & $3(1)$ & $10(3)$ & $<0.01$ \\
Loss to follow-up & $7(2)$ & $28(8)$ & Ref \\
Not evaluated & $21(6)$ & $21(6)$ & \\
Failure & $3(1)$ & $3(1)$ & \\
Treatment regimen changed & $3(1)$ & $3(1)$ & \\
Transferred out & $0(0)$ & $0(0)$ & \\
Treatment adherence & & 63 & \\
Missed $\leq 2$ doses per month & 42 & 60 & \\
$\begin{array}{c}\text { Missed }>2 \text { doses } \\
\text { consecutively per month }\end{array}$ & 30 & 3.3 & \\
$\begin{array}{c}\text { Non-adherence rate } \\
\text { (per 100 person-months) }\end{array}$ & 1.5 & & \\
\hline
\end{tabular}

*Note: Chi-square test "p-value".

Box 1. Key challenges encountered by the investigators during the development of software and implementation process.

\section{Challenges}

1) During development of software application

- Frequent change in RNTCP programme guidelines

- Complex mechanisms for treatment monitoring

- Minimal knowledge of health systems by software developers

- Software compatibility issues with android mobile phones from different companies available in the market

- Application could not be placed in "Google play store" for security issues

2) During Implementation process

- Compatibility issues with mobile application

- Change in mobile numbers of patients and health staff

- Loss of mobile handsets by patients

- Accidental deletion of mobile application in phones

- Internet connectivity issues

- Draining of mobile battery charge

\section{Discussion}

To our knowledge, this is one of the few studies conducted in India to determine the effectiveness of patient-centric mobile application to monitor tuberculosis patient treatment real-time under programmatic settings. The major findings of the study are that there was reduction in loss to follow-up rate ( $2 \%$ vs $8 \%)$ and increase in treatment success rate ( $89 \%$ vs $81 \%$ ) though not statistically significant among patients without mobile application. The non-adherence rate per 100 person-months was reduced by $50 \%$ among patients with mobile application.

The strengths of the study are 1) the study was conducted under routine pro- 
grammatic settings with a representative sample of patients from the district, 2) the findings of the intervention group are compared with the non-intervention group, 3) the challenges faced during development and implementation of the mobile application are elucidated. The limitations of the study are: 1) The study was conducted only in one district of Karnataka. The study findings may not be generalizable to other districts in the country because the Bangalore city is better performing programmatically and has extensively urban population. 2) Due to the resource constraints, we did not interview the patients to understand their perspective on having the mobile application as an additional tool to monitor their treatment. 3) We could not ascertain the patients drug intake as recorded through the mobile application as "drug taken" by testing their urine samples for isoniazid. 4) The patients selected for the intervention was based on convenient sampling. The patients were asked whether they have smart phones with internet connection and were willing to be part of the study. Only such patients diagnosed at public health facilities were included in the study. 5) We collected the data on the demographic and clinical variables of the patients that are routinely documented under the programme. We did not collect the information on several other variables such as socio-economic status, educational status that are known to influence the patient's adherence to treatment on having mobile application. We are unable to account for the influence of these variables on our study results.

Despite these limitations, the study has following programmatic implications. Firstly, the TB drug reminder mobile application augments the patient's treatment adherence in addition to the existing treatment monitoring mechanisms. The non-adherence rate per 100 persons-months is reduced by $50 \%$ and the loss-to follow-up rate is reduced when compared to those without mobile application. Several studies have shown that electronic reminders improve adherence to chronic medication [8]. Patient-centric mobile application and providing human touch to patients through directly observed treatment will strengthen the bonding between the patients and the health system and might improve treatment outcomes. It is implicit to have additional systems for patient monitoring in a vast country like India with limited available human resources under general health system [6]. Secondly, there were implementation challenges as seen by the investigators. They were 1) all the mobile phones were not compatible with the application, 2) change of mobile numbers among patients while on treatment halted the monitoring process, 3 ) draining of battery charge in the mobiles after installing mobile application was a issue among patients and, 4) the strength of mobile internet connectivity was a constraint to constantly sync the drug intake in the mobile application with the web based application which were used by the programme managers in the background to monitor all the patients with mobile application, 5) it was also found that the usage of smart phones among older population was comparatively less. Hence, we opine that the programme should develop an efficient and robust mobile application on similar lines for patients 
and also support the patients by providing a mobile phone with application during their treatment period wherever deemed necessary [12] [13].

\section{Conclusion}

To conclude, the findings from our study suggest that patient-centric mobile technology for adherence along with the existing monitoring mechanisms under the programme reduces the missed doses and contributes towards better treatment outcomes similar to the other digital adherence technologies [3] [11] [14]. The programme should adopt such technologies at a faster pace in pursuit to realize the dream to End-TB in India by 2025.

\section{Acknowledgements}

We acknowledge the support of all the TB patients who have participated in the study. Our special thanks to RNTCP staff of Bangalore city and officials from State TB office, Karnataka for their guidance for the conduct of the study. We are grateful to the Advanced Research Centre, Rajiv Gandhi University of Health Sciences (RGUHS), Karnataka for their financial support to conduct the study.

\section{Funding}

The research project was funded by the Rajiv Gandhi University of Health Sciences (RGUHS), Bengaluru, Karnataka, India (M81: 2015-16). The funders had no role in study design, data collection and analysis or preparation of the manuscript.

\section{Authors Contributions}

- Protocol development: SBN, NK, NM.

- Data collection, entry and interpretation: SBN, NK, NM, DM, NS, SMK, AD.

- Manuscript drafting: SBN, NK, NM, AS.

- Manuscript review and approval: All investigators.

\section{Conflicts of Interest}

The authors declare no conflicts of interest regarding the publication of this paper.

\section{References}

[1] World Health Organization (2019) WHO Global TB Report 2019.

[2] Central TB Division (2019) M. India TB Report 2019.

[3] Linn, A.J., Vervloet, M., van Dijk, L., Smit, E.G. and Van Weert, J.C.M. (2011) Effects of e-Health Interventions on Medication Adherence: A Systematic Review of the Literature. Journal of Medical Internet Research, 13, e103. https://doi.org/10.2196/jmir.1738

[4] Vervloet, M., Linn, A.J., van Weert, J.C.M., de Bakker, D.H., Bouvy, M.L. and van Dijk, L. (2012) The Effectiveness of Interventions Using Electronic Reminders to 
Improve Adherence to Chronic Medication: A Systematic Review of the Literature. Journal of the American Medical Informatics Association, 19, 696-704. http://www.ncbi.nlm.nih.gov/pubmed/22534082 https://doi.org/10.1136/amiajnl-2011-000748

[5] Lester, R., Park, J.J., Bolten, L.M., Enjetti, A., Johnston, J.C., Schwartzman, K., et al. (2019) Mobile Phone Short Message Service for Adherence Support and Care of Patients with Tuberculosis Infection: Evidence and Opportunity. Journal of Clinical Tuberculosis and Other Mycobacterial Diseases, 16, Article ID: 100108.

http://www.ncbi.nlm.nih.gov/pubmed/31720432

https://doi.org/10.1016/j.jctube.2019.100108

[6] DiStefano, M.J. and Schmidt, H. (2016) mHealth for Tuberculosis Treatment Adherence: A Framework to Guide Ethical Planning, Implementation, and Evaluation. Global Health: Science and Practice, 4, 211-221.

http://www.ghspjournal.org/lookup/doi/10.9745/GHSP-D-16-00018 https://doi.org/10.9745/GHSP-D-16-00018

[7] Liu, X., Lewis, J.J., Zhang, H., Lu, W., Zhang, S., Zheng, G., et al. (2015) Effectiveness of Electronic Reminders to Improve Medication Adherence in Tuberculosis Patients: A Cluster-Randomised Trial. PLOS Medicine, 12, e1001876.

https://dx.plos.org/10.1371/journal.pmed.1001876

https://doi.org/10.1371/journal.pmed.1001876

[8] Alipanah, N., Jarlsberg, L., Miller, C., Linh, N.N., Falzon, D., Jaramillo, E., et al. (2018) Adherence Interventions and Outcomes of Tuberculosis Treatment: A Systematic Review and Meta-Analysis of Trials and Observational Studies. PLoS Medicine, 15, e1002595. https://doi.org/10.1371/journal.pmed.1002595

[9] Central TB Division Ministry of Health \& Family Welfare Government of India and World Health Organization (2014) Standards for TB Care in India. World Health Organization, New Delhi.

[10] Thekkur, P., Kumar, A.N., Chinnakali, P., Selvaraju, S., Bairy, R., Singh, A.R., et al. (2019) Outcomes and Implementation Challenges of Using Daily Treatment Regimens with an Innovative Adherence Support Tool among HIV-Infected Tuberculosis Patients in Karnataka, India: A Mixed-Methods Study. Global Health Action, 12, Article ID: 1568826. http://www.ncbi.nlm.nih.gov/pubmed/30712507 https://doi.org/10.1080/16549716.2019.1568826

[11] Subbaraman, R., de Mondesert, L., Musiimenta, A., Pai, M., Mayer, K.H., Thomas, B.E., et al. (2018) Digital Adherence Technologies for the Management of Tuberculosis Therapy: Mapping the Landscape and Research Priorities. BMJ Global Health, 3, e001018. http://www.ncbi.nlm.nih.gov/pubmed/30364330 https://doi.org/10.1136/bmjgh-2018-001018

[12] Agins, B.D., Ikeda, D.J., Reid, M.J.A., Goosby, E., Pai, M. and Cattamanchi, A. (2019) Improving the Cascade of Global Tuberculosis Care: Moving from the "What" to the "How" of Quality Improvement. The Lancet Infectious Diseases, 19, e437-e443. https://doi.org/10.1016/S1473-3099(19)30420-7

[13] Lönnroth, K. and Raviglione, M. (2015) The WHO's New End TB Strategy in the Post-2015 Era of the Sustainable Development Goals. Transactions of the Royal Society of Tropical Medicine and Hygiene, 110, 148-150.

https://doi.org/10.1093/trstmh/trv108

[14] Pop-Eleches, C., Thirumurthy, H., Habyarimana, J.P., Zivin, J.G., Goldstein, M.P., de Walque, D., et al. (2011) Mobile Phone Technologies Improve Adherence to Antiretroviral Treatment in a Resource-Limited Setting: A Randomized Controlled 
Trial of Text Message Reminders. AIDS, 25, 825-834.

https://insights.ovid.com/crossref?an $=00002030-201103270-00013$

https://doi.org/10.1097/QAD.0b013e32834380c1 